\title{
The Implication of Dropping Race from the MDRD Equation to Estimate GFR in an African American-Only Cohort
}

\author{
Ernie Yap iD, Yelyzaveta Prysyazhnyuk, Jie Ouyang, Isha Puri, Carla Boutin-Foster, \\ and Moro Salifu
}

Department of Medicine, SUNY Downstate Health Sciences University, Brooklyn, NY, USA

Correspondence should be addressed to Ernie Yap; ernie.yap@downstate.edu

Received 27 April 2021; Revised 2 June 2021; Accepted 28 October 2021; Published 16 November 2021

Academic Editor: Joshua Rein

Copyright (c) 2021 Ernie Yap et al. This is an open access article distributed under the Creative Commons Attribution License, which permits unrestricted use, distribution, and reproduction in any medium, provided the original work is properly cited.

\begin{abstract}
The widely used Modification of Diet in Renal Disease (MDRD) formula adapts a 1.212 multiplier for individuals who are identified as African Americans (AAs) or Blacks, which leads to a higher GFR estimation. As it stands, AAs have a lower prevalence of chronic kidney disease (CKD) but higher incidence of end-stage renal disease (ESRD) compared with Whites. Many hypotheses have been postulated to explain this paradox, but the imprecision of the GFR estimation with race-adaptation could be contributory. We performed a single-center, longitudinal, retrospective study on a cohort of outpatient AA patients using the MDRD and MDRD race removed and CKD-EPI and CKD-EPI race removed and their progression to CKD G5 (eGFR $<15 \mathrm{ml} / \mathrm{min} /$ $1.73 \mathrm{~m}^{2}$ ). 327 patients were analyzed. Median follow-up was 88.1 months (interquartile range, 34.4-129.1). When race was removed from MDRD, 39.9\% of patients in CKD G1/2 were reclassified to CKD G3a, 72.6\% of patients in CKD G3a would be reclassified to CKD G3b, and 54.1\% and 36.4\% of patients would be reclassified from CKD 3b to CKD G4 and CKD G4 to CKD G5, respectively $(p<0.0001)$. Comparing the CKD-EPI formula against the MDRD in our cohort, we found that $8.2 \%, 18.8 \%$, and $11.4 \%$ of patients were reclassified from CKD G1/2 to CKD G3a, CKD G3a to G3b, and CKD G3b to CKD G4 respectively. Overall median time to progression to CKD G5 was 137.4 (131.9-142.8) months in patients who were not reclassified and 133.6 (127.6-139.6) months for patients who were reclassified by $\operatorname{MDRD}_{\text {race removed }}(p<0.288)$. Concerns of inequitable access to healthcare have elicited calls to review race-corrected eGFR equations. A substantial number of individuals would have their CKD stage reclassified should have the $\mathrm{MDRD}_{\text {race removed }}$ equation be adopted en masse on an AA-only population. The discrepancy is highest at the $45-59$ and $>60 \mathrm{ml} / \mathrm{min} / 1.72 \mathrm{~min}^{2}$ ranges. This will have tremendous impact on our center's approach to pharmacological dosing, referral system, best practices, and outcome surveillance. Comprehensive review of the current "racecorrected" eGFR will require a multifaceted approach and adjunctive use of noncreatinine-based approach.
\end{abstract}

\section{Introduction}

Chronic kidney disease (CKD) is defined as abnormalities of kidney structure or function, present for more than 3 months, with adverse implications on health. Equations that determine the estimated filtration rate (eGFR) are used universally to determine the CKD stage and guide clinical decision-making, including appropriate drug dosing, dialytic therapies, or listing for kidney transplantation. African Americans (AA) have lower prevalence of CKD [1] but higher incidence of end-stage renal disease (ESRD) compared with Whites [2]. The use of eGFR may contribute to the discrepancy between the prevalence of CKD and ESRD because these equations result in a higher reported eGFR for anyone identified as AA.

While studies have observed a higher serum creatinine in AA with CKD [3], the association of serum creatinine is not fully explained by body composition. The inclusion of race in the estimation of GFR is controversial and calls for further attention. Despite a lack of scientific evidence supporting racial genetic clusters and that race is a social construct, race as a variable continues to be inserted in biological measures [4]. Race has been integrated in the interpretation of pulmonary function, determination of need for bone mass density in Black women, and optimal weight for assessing metabolic risks in Asian adults [5]. 
The rationale for the use of race as a coefficient in eGFR equations is that it mitigates bias and improves performance of the equation on the population from which it is derived $[6,7]$. However, this assertion was based on the assumption that all AA have higher muscle mass, which was not based on actual measurement of muscle mass [8]. As an example, the inclusion of race would consider muscle mass of a patient with muscular dystrophy equal in contribution to GFR of a person with myostatin-related muscle hypertrophy, a rare condition of reduced body fat and increased skeletal muscle size, if both were AA [9].

Accurate staging of $\mathrm{CKD}$ is critically important in medical care and tremendous impact in raising awareness for the individual patient and implementation of key primary and secondary preventive measures such as control of hypertension, the use of renin-angiotensin-aldosterone inhibition, sodium-glucose-transporter inhibitor therapy, and proper medication dosing [10].

The use of race does not account for heterogeneity within racial groups. Therefore, the use of a race coefficient in the estimation of diagnostic biological measures is inherently flawed and devoid of a strong scientific premise and lacks precision [8]. The inclusion of "race-corrected" eGFR essentially overestimates GFR in a large part of the population and leads to missed opportunities for early intervention, referral to treatment, and as has been shown recently, access to kidney transplantation [11]. Re-examination of GFR estimating equations within predominant AA cohorts is critical to eliminating racial inequities in $\mathrm{CKD}$ outcomes $[12,13]$.

A cross-sectional study involving 2225 AA patients showed that $33.4 \%$ of them would be hypothetically reclassified to a more severe CKD stage if the race multiplier was removed from the CKD-EPI equation [14]. In an analysis of the National Health and Nutrition Examination Survey (NHANES) data, it was found that removal of the race adjustment increased CKD diagnoses among AAs (14.9\% to $18.4 \%$ (95\% CI, $3.2 \%-3.9 \%)$ ) and would have enhanced access to specialist care to these individuals. However, the authors conceded that many institutions use the Modification of Diet in Renal Disease (MDRD) equation and posited that elimination of the race correction could lead to even more individuals being reclassified due to the higher race-coefficient in MDRD [15].

The objective of this study is to determine the impact of removing the race correction from the MDRD and CKD-EPI on CKD staging and analyze the progression to CKD 5 $\left(\mathrm{eGFR}<15 \mathrm{ml} / \mathrm{min} / 1.73 \mathrm{~m}^{2}\right)$ in people who are reclassified by the correction removal.

\section{Methods}

2.1. Patients and CKD Stratification. A retrospective, longitudinal review of the electronic health record (EHR) of Nephrology AA patients from a large academic medical center located in Central Brooklyn was performed. Our center serves a predominantly AA community that has been subject to widespread disparate health outcomes [16]. The race/ethnicity makeup is as follows: White-3\%,
Black-86\%, Hispanic-8\%, and Asian-2\% [17]. Residents in this community have higher rates of comorbid conditions such as hypertension, diabetes, and obesity which places them at higher risk for developing CKD. The community as a whole also has a higher poverty rate, rent burden, and uninsured individuals compared to the rest of New York City [18]. Race designation was obtained from the EHR which is a mixture of self-identification and administrative designation.

For all patients, the laboratory measurement of serum creatinine was performed using the Beckman Coulter AU2700 system (Beckman Coulter, Inc., Brea, California, USA). Serum creatinine was analyzed by the kinetic alkaline picrate methodology which is traceable to the reference method based on isotope dilution-mass spectrometry (IDMS). GFR was estimated according to the MDRD abbreviated formula $[6,19]$.

We used the 2012 KDIGO Clinical Practice Guideline for the Evaluation and Management of CKD, which classifies estimated GFR in the following ranges:

(i) Stage G1/2: $-60-89 \mathrm{ml} / \mathrm{min} / 1.73 \mathrm{~m}^{2}$

(ii) Stage G3a: 45 to $59 \mathrm{ml} / \mathrm{min} / 1.73 \mathrm{~m}^{2}$

(iii) Stage G3b: 30 to $44 \mathrm{ml} / \mathrm{min} / 1.73 \mathrm{~m}^{2}$

(iv) Stage G4: 15 to $29 \mathrm{ml} / \mathrm{min} / 1.73 \mathrm{~m}^{2}$

(v) Stage G5: less than $15 \mathrm{ml} / \mathrm{min} / 1.73 \mathrm{~m}^{2}$ [20]

The following equations are used:

(i) MDRD: eGFR $\left(\mathrm{ml} / \mathrm{min} / 1.73 \mathrm{~m}^{2}\right)=175 \times\left(\mathrm{S}_{\mathrm{Cr}} / 88.4\right)^{-}$ ${ }^{1.154} \times$ age $^{-0.203} \times 1.212$ (if the patient is $\mathrm{AA}$ ) $\times 0.742$ (if female) [21].

(ii) $\mathrm{MDRD}_{\text {race }}$ removed: $\quad \mathrm{eGFR}=175 \times\left(\mathrm{S}_{\mathrm{Cr}} / 88.4\right)^{-}$ ${ }^{1.154} \times$ age $^{-0.203} \times 0.742$ (if female)

(iii) CKD-EPI: eGFR $=141 \times \min \left(\mathrm{S}_{\mathrm{Cr}} / \kappa, 1\right)^{\alpha} \times \max \left(\mathrm{S}_{\mathrm{Cr}} /\right.$ $\kappa, 1)^{-1.209} \times 0.993^{\text {Age }} \times 1.018$ (if female) $\times 1.159$ (if AA) [22].

(iv) CKD-EPI $\mathrm{I}_{\text {race removed }} \mathrm{eGFR}=141 \times \min \left(\mathrm{S}_{\mathrm{Cr}} / \kappa, 1\right)^{\alpha} \times$ $\max \left(\mathrm{S}_{\mathrm{Cr}} / \kappa, 1\right)^{-1.209} \times 0.993^{\text {Age }} \times 1.018$ (if female)

2.2. Baseline Data. Past medical history of hypertension, diabetes mellitus, congestive heart failure, stroke, coronary artery disease, and dyslipidemia and etiology of CKD and laboratory data were extracted from the EHR which is populated during in-person medical visits. The body mass index (BMI) was measured by calculating the individual's weight divided by the square of the height and expressed as $\mathrm{kg} / \mathrm{m}^{2}$.

2.3. Outcome Data. The observation period of each patient was defined as the time period from the first registered measurement of serum creatinine until CKD G5, defined as $\mathrm{eGFR}<15 \mathrm{ml} / \mathrm{min} / 1.73 \mathrm{~m}^{2}$.

2.4. Statistical Analyses. Continuous variables are presented as mean $\pm \mathrm{SD}$. Comparison of continuous variables between stages of CKD were performed using one-way ANOVA or the Kruskal-Wallis test, depending on the underlying 
distribution. Categorical and nominal data were compared using the $\mathrm{X}^{2}$ test. The Kaplan-Meier method was used to describe the progression to CKD G5. The log-rank test was used to compare between groups [23]. All computations were performed with SPSS 27. Data collection protocol was approved by the Research and Ethics Committee of SUNY Downstate Medical Center (no. 1516654-2).

\section{Results}

3.1. Demographic and Clinical Characteristics. 327 AA patients were analyzed. The mean age of the cohort was $61.9 \pm 14.2$ years. $60 \%$ of patients were female, and $57.5 \%$ had a diagnosis of diabetes mellitus. Body mass index (BMI) was evenly distributed across all groups. Over $90 \%$ of patients had a diagnosis of hypertension across all eGFR categories (Table 1).

3.2. Patient Outcomes. Median follow-up was 88.1 months (interquartile range, 34.4-129.1). Table 2 shows the change in the number of patients in their respective CKD stages in which $\mathrm{MDRD}_{\text {race removed }}, \mathrm{CKD} \mathrm{EPI}$, and CKD EPI $\mathrm{I}_{\text {race removed }}$ are applied, with the number of patients in CKD G3b almost doubling at the expense of the earlier stages in $M D R D_{\text {race }}$ removed compared to MDRD.

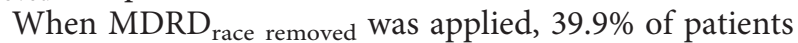
in CKD 1/2 were reclassified to CKD G3a, $71.8 \%$ of patients in CKD G3a would be reclassified to CKD 3b, and $54.1 \%$ and $36.4 \%$ of patients would be reclassified from CKD G3b to CKD G4 and CKD G4 to CKD G5, respectively $(p<0.0001)$ (Table 3). When the CKD-EPI formula was applied against the MDRD in our cohort, we found that $8.2 \%, 18.8 \%$, and $11.4 \%$ of patients were reclassified from CKD G1/2 to CKD G3a, CKD G3a to G3b, and CKD G3b to CKD G4, respectively, and a total of 3 patients were reclassified from CKD G4 to CKD G5 $(p<0.0001)$ (Table 4$)$.

To further evaluate the impact of removing race multiplier which our laboratory adopted the CKD-EPI formula, we compared it with CKD-EPI $\mathrm{I}_{\text {race removed }}$ and found that CKD stage reclassification is less severe but remained significant with $22.6 \%, 46.5 \%$, and $38.3 \%$ of patients reclassified from CKD G1/2, G3a, and G3b, respectively $(p<0.0001)$ (Table 5).

The time to progression to CKD was compared with the same patients when staged by MDRD with race compared to MDRD race removed. The Kaplan-Meier curves for the end point of reaching eGFR $<15 \mathrm{ml} / \mathrm{min} / 1.73 \mathrm{~m}^{2}$ (CKD G5) in patients with different stages of CKD who were not reclassified by $M D R D_{\text {race-removed }}$ and those who were reclassified are depicted in Figures 1 and 2, respectively. Overall median time to progression to CKD G5 was 137.4 (131.9-142.8) months in patients who were not reclassified and 133.6 (127.6-139.6) months for patients were reclassified after removal of race $(p<0.288)$. Advanced age was a significant predictor of progression to CKD G5 (Table 6). Overall median time to CKD G5 for all patients was 135 (131.6-139.6) months (Supplementary Figure 1).

\section{Discussion}

Our analysis demonstrated the impact of removing race from MDRD and CKD-EPI equations in an AA-only patient population. In both formulas, when the race multiplier was removed, a substantial number of patients would be reclassified to higher $\mathrm{CKD}$ stages. Patients who were reclassified were significantly older and trended towards a more rapid progression to CKD G5.

Our results are in line with the works of Ahmed et al. [14] and Diao et al. [15] who reported similar reclassification rates from larger datasets. On an intuitive level, this finding is not surprising in which removal of a positive multiplier would result in a smaller mathematical numeral. However, in our study, we showed that patients who were reclassified by $M D R D_{\text {race removed }}$ were significantly older in comparison with those who were not (Table 6), yet the median time to CKD G5 was not significantly shorter than their younger counterparts. This affirms the role of age in the eGFR equations and contributes to the ongoing, no-less profound debate on CKD-reclassification with respect to age; whether some people having lower renal mass as part of the normal aging process should be regarded as suffering from CKD [24]. It also implies that inclusion of race in the estimation of GFR may have greater impact on older adults. As well, in our cohort, clinical predictors such as past medical history and etiology of CKD are not a significant influence of reclassification. Tellingly, a substantial percentage $(71.8 \%)$ of our patients would be reclassified from stage G3a (eGFR between 45 and $59 \mathrm{ml} / \mathrm{min} / 1.73 \mathrm{~m}^{2}$ ) to stage G3b (eGFR between 30 and $44 \mathrm{ml} / \mathrm{min} / 1.73 \mathrm{~m}^{2}$ ) (Table 3 ). In a study involving $1,120,295$ adults with eGFR classified by the MDRD equation, Go et al. found that risks of death, cardiovascular events, and hospitalization were increased sharply for people with eGFR less than $45 \mathrm{ml} / \mathrm{min} / 1.73 \mathrm{~m}^{2}$, denoting that the clinical importance of the accuracy is determining this cutoff [25].

Our hospital laboratory continues to report eGFR using the MDRD equation, which formed the foundation of this study. The MDRD study equation has been shown to be accurate in the low GFR range (CKD stages G3 and G4), but it underestimates GFR in higher GFR ranges (i.e., in healthy subjects) [26]. In recognition of the greater accuracy of CKD-EPI [27], due to its improvement in bias, we simulated CKD-EPI on our cohort, compared it with CKD-EPI race removed (Table 5), and showed lower reclassification rates across all eGFR ranges.

In a cohort of 533 healthy Black Africans using a cutoff of $75 \mathrm{ml} / \mathrm{min} / 1.73 \mathrm{~m}^{2}$, the 4 -variable MDRD equation without correction for race classified the highest proportion of participants under the age of 40 years $(7.8 \%)$ as having reduced eGFR [28]. In 2001, in recognition of the greater serum and urine creatinine levels amongst participants in the African American Study of Hypertension and Kidney Disease (AASK) cohort, Lewis et al. derived a five-variable eGFR formula [29]. However, the Lewis equations have never been used in clinical practice, likely due to subsequent widespread adoption of the MDRD formula. A study involving Black Africans from Ghana concluded that 
TABle 1: Patient characteristics.

\begin{tabular}{|c|c|c|c|c|c|c|}
\hline \multirow{2}{*}{ Characteristics } & \multirow{2}{*}{ Total $(N=327)$} & \multicolumn{4}{|c|}{ Chronic kidney disease glomerular stage by MDRD formula } & \multirow{2}{*}{$p$ value } \\
\hline & & $\mathrm{G} 1 / 2(N=183)$ & G3a $(N=85)$ & $\mathrm{G} 3 \mathrm{~b}(N=37)$ & $\mathrm{G} 4(N=22)$ & \\
\hline Age (year) & $61.9 \pm 14.2$ & $58.6 \pm 14.4$ & $65.7 \pm 12.5$ & $66.2 \pm 13.9$ & $68.1 \pm 11.5$ & 0.0002 \\
\hline Female sex $(\%)$ & 60.6 & 61.7 & 60.0 & 56.8 & 59.1 & 0.811 \\
\hline Body mass index $\left(\mathrm{kg} / \mathrm{m}^{2}\right)$ & $29.7 \pm 6.8$ & $29.9 \pm 7.0$ & $29.4 \pm 6.6$ & $30.8 \pm 6.4$ & $27.1 \pm 6.2$ & 0.218 \\
\hline $\mathrm{GFR}, \mathrm{ml} / \mathrm{min} / 1.73 \mathrm{~m}^{2}$ & $67.4 \pm 27.2$ & $86.2 \pm 19.8$ & $52.4 \pm 4.4$ & $37.0 \pm 4.3$ & $20.2 \pm 3.5$ & $<0.0001$ \\
\hline \multicolumn{7}{|l|}{ Medical history (\%) } \\
\hline Hypertension & 94.2 & 92.9 & 97.6 & 89.2 & 100 & 0.141 \\
\hline Diabetes & 57.5 & 57.4 & 55.3 & 73.0 & 40.9 & 0.099 \\
\hline Congestive heart failure & 19.3 & 19.7 & 21.2 & 16.2 & 13.6 & 0.828 \\
\hline Stroke & 13.5 & 15.8 & 8.2 & 8.1 & 22.7 & 0.143 \\
\hline Coronary artery disease & 24.5 & 24.6 & 22.4 & 35.1 & 13.6 & 0.274 \\
\hline Dyslipidemia & 63.3 & 60.7 & 67.1 & 64.9 & 68.2 & 0.721 \\
\hline \multicolumn{7}{|l|}{ Etiology of CKD (\%) } \\
\hline Hypertensive nephrosclerosis & 59.6 & 54.6 & 67.1 & 59.5 & 72.7 & 0.144 \\
\hline Diabetic kidney disease & 37.9 & 38.8 & 32.9 & 45.9 & 36.4 & 0.574 \\
\hline Glomerulonephritis & 7.6 & 9.8 & 5.9 & 2.7 & 4.5 & 0.362 \\
\hline Polycystic kidney disease & 4.3 & 4.4 & 3.5 & 2.7 & 9.1 & 0.662 \\
\hline Others & 24.2 & 26.8 & 22.4 & 21.6 & 13.6 & 0.514 \\
\hline
\end{tabular}

TABLE 2: CKD staging of patients according to the respective eGFR formulas from a cohort of 327 AA patients.

\begin{tabular}{lcccc}
\hline \multirow{2}{*}{ eGFR $\left(\mathrm{ml} / \mathrm{min} / 1.73 \mathrm{~m}^{2}\right)$} & \multicolumn{3}{c}{ Number of patients according to estimation formulas (\%) } \\
& MDRD & MDRD $_{\text {race removed }}$ & CKD EPI & CKD EPI race removed \\
\hline$>60$ & $183(56)$ & $110(33.6)$ & $168(51.4)$ & $130(39.8)$ \\
$45-60$ & $85(26)$ & $96(29.4)$ & $86(26.3)$ & $84(25.7$ \\
$30-45$ & $37(11.3)$ & $78(23.9)$ & $23(7)$. & $69(21.1)$ \\
$15-30$ & $22(6.7)$ & $35(10.7)$ & $3(0.9)$ & $37(11.3)$ \\
$<15$ & 0 & $8(2.4)$ & $7(2.1)$ \\
\hline
\end{tabular}

TABLE 3: Comparison between the MDRD and $M D R D_{\text {race removed }}$ formulas.

\begin{tabular}{|c|c|c|c|c|c|c|}
\hline & \multicolumn{6}{|c|}{$\mathrm{MDRD}_{\text {race removed }}\left(\mathrm{m} / \mathrm{min} / 1.73 \mathrm{~m}^{2}\right)$} \\
\hline \multirow{5}{*}{ MDRD eGFR $\left(\mathrm{m} / \mathrm{min} / 1.73 \mathrm{~m}^{2}\right)$} & & $>60$ & $45-59$ & $30-44$ & $15-29$ & $<15$ \\
\hline & $>60$ & 110 & 73 (39.9\%) & & & \\
\hline & $45-59$ & & 23 & $61(71.8 \%)$ & 1 & \\
\hline & $30-44$ & & & 17 & $20(54.1 \%)$ & \\
\hline & $15-29$ & & & & 14 & $8(36.4 \%)$ \\
\hline
\end{tabular}

TABLE 4: Comparison between the MDRD and CKD-EPI formulas.

\begin{tabular}{|c|c|c|c|c|c|c|}
\hline & & & CKD & $1 / \mathrm{min} / 1.73$ & & \\
\hline & & $>60$ & $45-59$ & $30-44$ & $15-29$ & $<15$ \\
\hline & $>60$ & 168 & $15(8.2 \%)$ & & & \\
\hline $\operatorname{MDRD}$ eGFR $\left(\mathrm{m} / \mathrm{min} / 1.73 \mathrm{~m}^{2}\right)$ & $45-59$ & & 69 & $16(18.8 \%)$ & & \\
\hline & $30-44$ & & 2 & 31 & $4(10.8 \%)$ & \\
\hline & $15-29$ & & & & 19 & $3(13.6 \%)$ \\
\hline
\end{tabular}

correction for race was unnecessary for both the MDRD and CKD-EPI equations [30]. This study compared eGFR with creatinine clearance, and the mean weight of the participants was $54.4 \mathrm{~kg}$. In a cohort of 100 Black South Africans in which eGFR was compared with measured GFR (mGFR), Deventer et al. concluded that the race correction in the MDRD equation resulted in a median positive bias of 13.1 (95\% CI 5.5 to 18.3$) \mathrm{ml} / \mathrm{min} / 1.73 \mathrm{~m}^{2}$, in comparison with a median bias of $1.9(95 \%$ CI 0.8 to 4.5$) \mathrm{ml} / \mathrm{min} / 1.73 \mathrm{~m}^{2}$ when the race factor was discarded [31]. It should be noted that the mean weight of participants of the above studies was $54.5 \mathrm{~kg}$ and $69.6 \mathrm{~kg}$, respectively. For comparison, the mean weight of 
TABLE 5: Comparison between the CKD-EPI and CKD-EPI $I_{\text {race-removed }}$ formulas.

\begin{tabular}{|c|c|c|c|c|c|c|}
\hline & & & CKD-EPI & oved $(\mathrm{ml} / \mathrm{mi}$ & & \\
\hline & & $>60$ & $45-59$ & $30-44$ & $15-29$ & $<15$ \\
\hline & $>60$ & 130 & $38(22.6 \%)$ & & & \\
\hline CKD-EPI eGFR $\left(\mathrm{ml} / \mathrm{min} / 1.73 \mathrm{~m}^{2}\right)$ & $45-59$ & & 46 & $40(46.5 \%)$ & & \\
\hline CKD-EPI eGFK (ml/min/l.73 $\left.\mathrm{m}^{-1}\right)$ & $30-44$ & & & 29 & $18(38.3 \%)$ & \\
\hline & $15-29$ & & & & 19 & $4(17.4 \%)$ \\
\hline & $<15$ & & & & & 3 \\
\hline
\end{tabular}

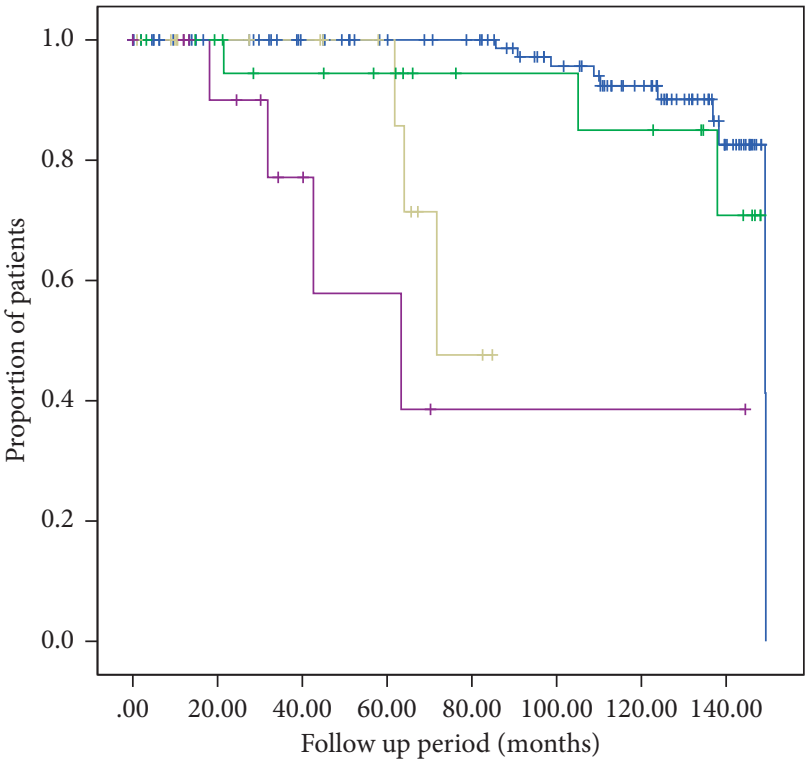

CKD stage

$\begin{array}{ll}\neg 1 & +1 \text {-censored } \\ \neg 2 & +2 \text {-censored } \\ \neg 3 & +3 \text {-censored } \\ \neg 4 & +4 \text {-censored }\end{array}$

FIgURE 1: Kaplan-Meier curves depicting median time to CKD G5 amongst AA patients who were not reclassified by $M D R D_{\text {race re- }}$ moved $(N=164)$.

participants in the AASK and MDRD cohorts was $89 \mathrm{~kg}$ and $79.6 \mathrm{~kg}$, respectively $[21,32]$. In a study that compared MDRD and CKD-EPI against mGFR in African Europeans originating from West Africa, a correction factor of 1.08 was derived [33]. Hence, the "race-correction" is in fact a reflection of assumptions of muscle mass, and consequently, the limitations surrounding the accuracy of these assumptions across swathes of population or even within an evolving population due to dietary changes will continue to hamper any formula's accuracy. Uribarri et al. recently showed that an oft-used clinical metric, the urine anion gap, can no longer be used reliably due to changes in diet across time resulting in variations to urine electrolytes [34].

In this study, the exclusion of race in both $M D R D_{\text {race }}$ removed and CKD-EPI race removed $_{\text {reclassified over } 49.5 \% \text { and }}$ $30.6 \%$ of our AA patients to a higher stage of CKD, respectively, in comparison with their conventional counterparts. This partially explains the seemingly paradoxical observation that although AA patients present with more

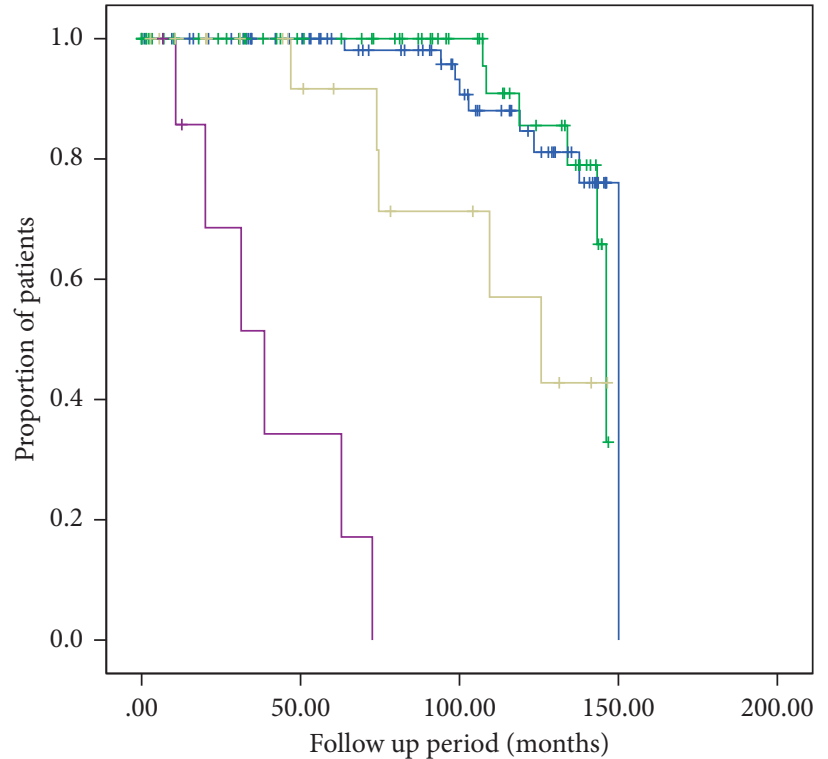

$$
\begin{array}{ll}
\text { CKD stage } & \\
\neg 1 & +1 \text {-censored } \\
\neg 2 & +2 \text {-censored } \\
\neg 3 & +3 \text {-censored } \\
\neg 4 & +4 \text {-censored }
\end{array}
$$

Figure 2: Kaplan-Meier curves depicting median time to CKD G5 amongst AA patients who were reclassified by MDRD race removed $(N=163)$.

advanced CKD stage than Whites, AAs on hemodialysis have a lower mortality than their White counterparts probably because once they are recognized within the healthcare system as dialysis patients, and eGFR becomes a less important determinant of healthcare resource [35]. Other factors such as neighborhood socioeconomic status have also been implicated [36].

Our study has the following limitations: we did not have mGFR, and as such, our comparison of various eGFR equations is not validated against a gold standard. Another major limitation is the absence of proteinuria data. As well, the definition of AA is a heterogenous one as our patient cohort comprises mainly Black individuals who derived their ancestry from the Caribbean [37]. As well, our dataset most likely suffers from lack of concordance between self-reported ethnicity and administrative race categorization due to the nature of EHRs [38, 39]. Given its retrospective nature, it is plausible that the progression to eGFR $<15 \mathrm{ml} / \mathrm{min} / 1.73 \mathrm{~m}^{2}$ has inherent biases. Thus, our results do not inform the 
TABLE 6: Comparison of AA patient characteristics of those were reclassified after removal of the race multiplier.

\begin{tabular}{|c|c|c|c|}
\hline \multirow{2}{*}{ Characteristics } & \multicolumn{2}{|c|}{ Chronic kidney disease glomerular stage by MDRD formula } & \multirow{2}{*}{$p$ value } \\
\hline & Patients who were reclassified & Patients who were not reclassified & \\
\hline Age (years) & $64.3 \pm 12.7$ & $59.6 \pm 15.2$ & 0.003 \\
\hline Female sex $(\mathrm{N})$ & 103 & 95 & 0.330 \\
\hline Body mass index $\left(\mathrm{kg} / \mathrm{m}^{2}\right)$ & $29.4 \pm 6.4$ & $29.9 \pm 7.2$ & 0.514 \\
\hline $\mathrm{GFR}\left(\mathrm{ml} / \mathrm{min} / 1.73 \mathrm{~m}^{2)}\right.$ & $52.5 \pm 14.3$ & $75.1 \pm 28.0$ & $<0.0001$ \\
\hline \multicolumn{4}{|l|}{ Past medical history $(\mathrm{N})$} \\
\hline Hypertension & 157 & 151 & 0.101 \\
\hline Diabetes & 94 & 94 & 0.949 \\
\hline Congestive heart failure & 34 & 29 & 0.467 \\
\hline Stroke & 21 & 23 & 0.762 \\
\hline Coronary artery disease & 46 & 34 & 0.115 \\
\hline Dyslipidemia & 104 & 103 & 0.851 \\
\hline \multicolumn{4}{|c|}{ Etiology of chronic kidney disease $(N)$} \\
\hline Hypertensive nephrosclerosis & 101 & 94 & 0.392 \\
\hline Diabetic kidney disease & 67 & 57 & 0.237 \\
\hline Glomerulonephritis & 10 & 15 & 0.306 \\
\hline Polycystic kidney disease & 4 & 10 & 0.104 \\
\hline Others & 34 & 45 & 0.165 \\
\hline
\end{tabular}

biological impact of removing the race correction in eGFR formulas but highlight the disparate classification that informs clinical decision-making paradigms in our center such as referral to specialists, eligibility for kidney transplantation, and other social and disability support.

In conclusion, the removal of race multiplier factor in eGFR formulas is highly impactful on CKD epidemiology and management. The identification of an adjunctive noncreatinine-based novel biomarker remains a critical unmet medical need.

\section{Data Availability}

The data used to support the findings of this study are available from the corresponding author upon request.

\section{Conflicts of Interest}

The authors declare that they have no conflicts of interest.

\section{Supplementary Materials}

Supplementary Figure 1. Kaplan-Meier curves depicting mean duration to progression to CKD 5 in all patients by CKD stage according to MDRD. (Supplementary Materials)

\section{References}

[1] J. Coresh, E. Selvin, L. A. Stevens et al., "Prevalence of chronic kidney disease in the United States," Jama, vol. 298, no. 17, pp. 2038-2047, 2007, (In eng).

[2] A. J. Collins, R. N. Foley, B. Chavers et al., "United States renal data system 2011 annual data report: atlas of chronic kidney disease \& end-stage renal disease in the United States," American Journal of Kidney Diseases: the Official Journal of the National Kidney Foundation, vol. 59, no. 1 Suppl 1, pp. e1-420, 2012, (In eng).

[3] J. Hsu, K. L. Johansen, C. Y. Hsu, G. A. Kaysen, and G. M. Chertow, "Higher serum creatinine concentrations in black patients with chronic kidney disease: beyond nutritional status and body composition," Clinical Journal of the
American Society of Nephrology, vol. 3, no. 4, pp. 992-997, 2008, (In eng).

[4] R. Yearby, "Race based medicine, colorblind disease: how racism in medicine harms us all," The American Journal of Bioethics, vol. 21, no. 2, pp. 19-27, 2021.

[5] J. P. Cerdeña, M. V. Plaisime, and J. Tsai, "From race-based to race-conscious medicine: how anti-racist uprisings call us to act," The Lancet, vol. 396, no. 10257, pp. 1125-1128, 2020.

[6] A. S. Levey, J. P. Bosch, J. B. Lewis, T. Greene, N. Rogers, and D. Roth, "A more accurate method to estimate glomerular filtration rate from serum creatinine: a new prediction equation," Annals of Internal Medicine, vol. 130, no. 6, pp. 461-470, 1999, (In eng).

[7] A. S. Levey, H. Tighiouart, S. M. Titan, and L. A. Inker, "Estimation of glomerular filtration rate with vs without including patient race," JAMA Internal Medicine, vol. 180, no. 5, pp. 793-795, 2020.

[8] V. Grubbs, "Precision in GFR reporting," Clinical Journal of the American Society of Nephrology, vol. 15, no. 8, pp. 1201-1202, 2020.

[9] K. R. Wagner and J. S. Cohen, Myostatin-Related Muscle Hypertrophy-Retired Chapter, for Historical Reference Only, University of Washington, Seattle, WA, USA, 1993.

[10] J. I. Shin, Y. Sang, A. R. Chang et al., "The FDA Metformin label change and racial and sex disparities in metformin prescription among patients with CKD," Journal of the American Society of Nephrology, vol. 31, no. 8, pp. 1847-1858, 2020, (In eng).

[11] L. R. Zelnick, N. Leca, B. Young, and N. Bansal, “Association of the estimated glomerular filtration rate with vs without a coefficient for race with time to eligibility for kidney transplant," JAMA Network Open, vol. 4, no. 1, p. e2034004, 2021.

[12] D. A. Vyas, L. G. Eisenstein, and D. S. Jones, "Hidden in plain sight-reconsidering the use of race correction in clinical algorithms," New England Journal of Medicine, vol. 383, no. 9, pp. 874-882, 2020.

[13] N. D. Eneanya, W. Yang, and P. P. Reese, "Reconsidering the consequences of using race to estimate kidney function," Jama, vol. 322, no. 2, pp. 113-114, 2019, (In eng). 
[14] S. Ahmed, C. T. Nutt, N. D. Eneanya et al., "Examining the potential impact of race multiplier utilization in estimated glomerular filtration rate calculation on african-american care outcomes," Journal of General Internal Medicine, vol. 36, no. 2, pp. 464-471, 2021, (In eng).

[15] J. A. Diao, G. J. Wu, H. A. Taylor et al., "Clinical implications of removing race from estimates of kidney function," JAMA, vol. 325 , no. 2, pp. 184-186, 2021.

[16] R. Gupta, R. Agrawal, Z. Bukhari et al., "Higher comorbidities and early death in hospitalized African-American patients with Covid-19," BMC Infectious Diseases, vol. 21, no. 1, p. 78, 2021 (In eng).

[17] U.S. Census Bureau, American Community Survey 5-year Estimates, NYC-rooklyn Community District 17-East Flatbush, Farragut \& Rugby PUMA, Brooklyn, NY, USA, 2019, http://censusreporter.org/profiles/79500US3604010-nyc-brook lyn-community-district-17-east-flatbush-farragut-rugby-puma -ny/.

[18] K. Hinterland, M. Naidoo, L. King et al., Community Health Profiles 2018, vol. 41, no. 59, pp. 1-20, Brooklyn Community District 17: East Flatbush, Brooklyn, NY, USA, 2018, https://www1.nyc.gov/assets/doh/downloads/pdf/ data/2018chp-bk17.pdf.

[19] A. S. Levey, T. Greene, J. W. Kusek, and G. J. Beck, “A simplified equation to predict glomerular filtration rate from serum creatinine," Journal of the American Society of $\mathrm{Ne}$ phrology, vol. 11, p. 155A, 2000 (Abstract).

[20] National Kidney Foundation, "K/DOQI clinical practice guidelines for chronic kidney disease: evaluation, classification and stratification," American Journal of Kidney Disorder, vol. 39, no. S1, p. s266, 2002.

[21] A. S. Levey, J. Coresh, T. Greene et al., "Using standardized serum creatinine values in the modification of diet in renal disease study equation for estimating glomerular filtration rate," Annals of Internal Medicine, vol. 145, no. 4, pp. 247-254, 2006, (In eng).

[22] A. S. Levey, L. A. Stevens, C. H. Schmid et al., "A new equation to estimate glomerular filtration rate," Annals of Internal Medicine, vol. 150, no. 9, pp. 604-612, 2009, (In eng).

[23] E. L. Kaplan and P. Meier, "Nonparametric estimation from incomplete observations," Journal of the American Statistical Association, vol. 53, no. 282, pp. 457-481, 1958.

[24] R. J. Glassock, P. Delanaye, and A. D. Rule, "Should the definition of CKD be changed to include age-adapted GFR criteria? YES," Kidney International, vol. 97, no. 1, pp. 34-37, 2020.

[25] A. S. Go, G. M. Chertow, D. Fan, C. E. McCulloch, and C. Y. Hsu, "Chronic kidney disease and the risks of death, cardiovascular events, and hospitalization," New England Journal of Medicine, vol. 351, no. 13, pp. 1296-1305, 2004.

[26] P. Delanaye and E. P. Cohen, "Formula-based estimates of the GFR: equations variable and uncertain," Nephron Clinical Practice, vol. 110, no. 1, pp. c48-c54, 2008, discussion c54. (In eng).

[27] P. Delanaye, H. Pottel, and R. Botev, "Con: should we abandon the use of the MDRD equation in favour of the CKDEPI equation?" Nephrology Dialysis Transplantation, vol. 28, no. 6, pp. 1396-1403, 2013.

[28] G. Omuse, D. Maina, J. Mwangi et al., "Comparison of equations for estimating glomerular filtration rate in screening for chronic kidney disease in asymptomatic black Africans: a cross sectional study," BMC Nephrology, vol. 18, no. 1, p. 369, 2017 (In eng).
[29] J. Lewis, L. Agodoa, D. Cheek et al., "Comparison of crosssectional renal function measurements in African Americans with hypertensive nephrosclerosis and of primary formulas to estimate glomerular filtration rate," American Journal of Kidney Diseases, vol. 38, no. 4, pp. 744-753, 2001, (In eng).

[30] J. B. Eastwood, S. M. Kerry, J. Plange-Rhule et al., “Assessment of GFR by four methods in adults in Ashanti, Ghana: the need for an eGFR equation for lean African populations," $\mathrm{Ne}$ phrology Dialysis Transplantation, vol. 25, no. 7, pp. 21782187, 2010, (In eng).

[31] H. E. van Deventer, J. A. George, J. E. Paiker, P. J. Becker, and I. J. Katz, "Estimating glomerular filtration rate in black South Africans by use of the modification of diet in renal disease and cockcroft-gault equations," Clinical Chemistry, vol. 54, no. 7, pp. 1197-1202, 2008, (In eng).

[32] J. T. Wright Jr., G. Bakris, and T. Greene, "Effect of blood pressure lowering and antihypertensive drug class on progression of hypertensive kidney diseaseresults from the AASK trial," Jama, vol. 288, no. 19, pp. 2421-2431, 2002, (In eng).

[33] M. Flamant, E. Vidal-Petiot, M. Metzger et al., "Performance of GFR estimating equations in African Europeans: basis for a lower race-ethnicity factor than in African Americans," American Journal of Kidney Diseases, vol. 62, no. 1, pp. 182-184, 2013, (In eng).

[34] J. Uribarri and M. S. Oh, "The urine anion gap: common misconceptions," Journal of the American Society of $\mathrm{Ne}$ phrology, vol. 32, no. 5, pp. 1025-1028, 2021, ASN.2020101509.

[35] J. A. Pugh, M. R. Tuley, and S. Basu, "Survival among Mexican-Americans, non-hispanic whites, and AfricanAmericans with end-stage renal disease: the emergence of a minority pattern of increased incidence and prolonged survival," American Journal of Kidney Diseases: the Official Journal of the National Kidney Foundation, vol. 23, no. 6, pp. 803-807, 1994, (In eng).

[36] T. S. Johns, M. M. Estrella, D. C. Crews et al., "Neighborhood socioeconomic status, race, and mortality in young adult dialysis patients," Journal of the American Society of $\mathrm{Ne}$ phrology, vol. 25, no. 11, pp. 2649-2657, 2014.

[37] Office of Management and Budget, Revisions to the Standards for the Classification of Federal Data on Race and Ethnicity, Executive Office of the President, Office of Management and Budget (OMB), Office of Information and Regulatory Affairs, Washington, DC, USA, 1997, https://www.govinfo.gov/ content/pkg/FR-1997-10-30/pdf/97-28653.pdf.

[38] E. V. Klinger, S. V. Carlini, I. Gonzalez et al., "Accuracy of race, ethnicity, and language preference in an electronic health record," Journal of General Internal Medicine, vol. 30, no. 6, pp. 719-723, 2015, (In eng).

[39] N. S. Hamilton, D. Edelman, M. Weinberger, and G. L. Jackson, "Concordance between self-reported race/ ethnicity and that recorded in a veteran Affairs electronic medical record," North Carolina Medical Journal, vol. 70, no. 4, pp. 296-300, 2009, (In eng). 